\title{
Simulador applet descartes: como didáctica de enseñanza de la función cuadrática
}

\section{Descartes applet simulator: as a teaching didactic of the quadratic function}

Jorge Enrique Díaz Pinzón

Consultor Independiente, Ecuador

Autor para correspondencia: jorgediaz333@gmail.com

Fecha de recepción: 11 de Mayo de 2017 - Fecha de aceptación: 10 de Agosto de 2017

Resumen: El proyecto se realizó mediante un tipo de estudio exploratorio, pues presenta una visión general y aproximada del objeto de estudio, cuando un tema ha sido poco explorado. Además de contar una investigación de tipo cuantitativo. Se indago sobre el uso del software matemático Applet Descartes versión 3.815 como estrategia didáctica en la enseñanza de la función cuadrática, en los estudiantes de grado 904 de la Institución Educativa General Santander.Para tal fin de la investigación se utilizaron encuestas directas de carácter individual y de acuerdo a su forma de tipo cerrada. Estás fueron aplicadas como una técnica que utiliza un cuestionario con diversos tipos de preguntas con el fin de conseguir mediciones cuantitativas sobre algunas características objetivas y subjetivas de la población, que después ser sistematizadas, analizadas e interpretadas se observó por parte de los estudiantes como la implementación del software matemático Applet descartes versión 3.815, sirvió como una estrategia de enseñanza y de este modo contactar a los estudiantes de grado noveno con las nuevas tecnologías de la información y la comunicación -TIC-Como el computador y el Internet y profundizar en los contenidos relacionados con la función cuadrática.

Palabras clave: applet descartes; simulador; función cuadrática

Abstract: The project was carried out by a type of exploratory study; it presents a general and approximate view of the object of study, when a topic has been little explored. In addition to quantitative research. I inquired about the use of mathematical software version 3.815 Applet Descartes as a teaching strategy in teaching the quadratic function, 904 undergraduate students of School General Santander.To this end the research used direct surveys of individual and according to their form of closed type. You were applied as a technique using a questionnaire with different types of questions in order to get quantitative measurements on some objective and subjective characteristics of the population, which then be systematized, analyzed and interpreted was observed by students as the implementation Descartes applet mathematical software version 3815, served as a teaching strategy and thereby placing ninth grade students in touch with the new technologies of information and communication, TIC, such as computers and the internet and deepen the standards related content quadratic function.

Key words: applet discards; simulator; quadratic function 


\section{Introducción}

El avance del pensamiento espacial y las representaciones de figuras y gráficos son significativos para el currículo de las matemáticas escolares, lo cual se reafirma en los lineamientos curriculares de matemáticas donde se reconoce la relevancia de volver a recuperar el sentido espacial intuitivo en toda la matemática.

De acuerdo con Díaz (2016) "La mayoría de docentes Institución Educativa General Santander, no cuentan con una alternativa extra- aula para desarrollar procesos educativos con miras a generar aprendizajes significativos que sirvan de modelo al resto de la comunidad educativa".

En el presente proyecto, se desarrolló el proceso de implementación y evaluación de un software educativo para facilitar y mejorar el proceso de enseñanza y de aprendizaje de un tema concerniente a la función cuadrática, considerando que la Informática en la Educación, sobre todo en la Educación Matemática, es un medio poderoso para desarrollar en el estudiante sus potencialidades, creatividad e imaginación. Además de nuevos ambientes de aprendizaje que le permitan al estudiante generar un gusto por el conocimiento. Bagur (2011).

\section{Marco teórico}

Según informe Mundial sobre la Educación de la UNESCO (2012), "El Imperativo de la Calidad, enfatizó en la importancia de los métodos de aprendizaje y en la utilización de materiales educativos, infraestructura y acceso a las TIC, como un importante desafío en el campo educativo".

Según FIB ( 2008). "La historia y la evolución de la simulación por ordenador han ido paralelas a la evolución de la Informática".

De otra parte Cuevas ( 2000). “Cuesta mucho tiempo y esfuerzo desarrollar software para implementarlos en actividades educativas dentro de la matemática, ya que al tratar de enseñar a los alumnos un concepto matemático se debe presentar la reunión de varios mundos, contextos o registros de representación semiótica"

Según Pizarro (2009) "las teorías, que son el soporte para las formas de concebir el proceso de enseñanza aprendizaje, pueden dar forma a nuevos métodos pedagógicos. En definitiva, el poder de las Tics para crear nuevos y atractivos ámbitos de aprendizaje para los estudiantes, estará dado por la habilidad de los docentes en el uso de estas herramientas”

Segú relaciona el MEN (2003)“Las competencias matemáticas no se alcanzan por generación espontánea, sino que requieren de ambientes de aprendizaje enriquecidos por situaciones problemas significativos y comprensivos"

Esto en concordancia con McFarlane (1999). "Entendemos que denota el software que se utiliza en un contexto educativo, es un término que abarca una variedad amplia y ecléctica de herramientas y recursos. De hecho, engloba un conjunto de entidades tan variables que el hecho 
de depender de un entorno informatizado crea una impresión de homogeneidad que no resiste un análisis meticuloso"

Ahora bien Mariño (2008). "Las expectativas que crea el computador como medio de enseñanza-aprendizaje se fundamentan tanto en las características técnicas que tiene la máquina como en los desarrollos de la tecnología educativa en que se fundamenta el diseño de ambientes de aprendizaje."

\section{Applet descartes versión 3.815}

Según Muñoz (2013). "Descartes es una herramienta (software libre) que permite de forma sencilla diseñar a los usuarios sus propios recursos didácticos a través de escenas animadas interactivas, que pueden ser utilizadas en cualquier área o materia del currículum educativo."

Del mismo modo Muñoz (2013). "Se llama Applet a un pequeño programa que puede incluirse en páginas web, realizado en un lenguaje de programación denominado Java.

Descartes es un Applet configurable, y cada configuración de Descartes da lugar a un programa interactivo diferente que se llama escena".

\section{Función Cuadrática}

Según (Fatela.M, 2009) "La función cuadrática es una función muy común en Matemática. Se trata de una función de segundo grado: la "x" aparece elevada al cuadrado como máxima potencia".

\section{Metodología de la investigación}

El proyecto se realizó mediante un tipo de estudio exploratorio, pues presenta una visión general y aproximada del objeto de estudio, cuando un tema ha sido poco explorado. Además de contar una investigación de tipo cuantitativo.

Según Hurtado y Toro (1998) citado por Salinas (2010).La investigación Cuantitativa: "La investigación cuantitativa tiene una concepción lineal, es decir que haya claridad entre los elementos que conforman el problema, que tenga definición, limitarlos y saber con exactitud cómo se inicia el problema, también es importante saber qué tipo de incidencia existe entre sus elementos".

La población según Balestrini (2006), se define como "cualquier conjunto de elementos de la que se quiere conocer o investigar alguna de sus características".

Este proyecto se centra en estudiantes de la jornada de la tarde, específicamente con estudiantes grado noveno cuyas edades oscilan entre los 13 y 16 años.

\section{Instrumentos de Investigación}




\section{Encuesta}

Para la investigación se utilizó el instrumento de recolección de la información mediante encuesta. Según Huamán (2005) "La encuesta es una técnica destinada a obtener datos de varias personas cuyas opiniones impersonales interesan al investigador”.

Las preguntas cerradas consisten en proporcionar al sujeto observado una serie de opciones para que escoja una como respuesta. Tienen la ventaja de que pueden ser procesadas más fácilmente y su codificación se facilita.

Para la investigación se utilizaron encuestas directas de carácter individual y de acuerdo a su forma de tipo cerrada. Estás fueron aplicadas como una técnica que utiliza un cuestionario con diversos tipos de preguntas con el fin de conseguir mediciones cuantitativas sobre algunas características objetivas y subjetivas de la población.

\section{Encuesta diagnóstica}

Se llevó a cabo una encuesta diagnóstico el día 9 de junio de 2013, a 40 estudiantes de grado 904 JT, de la I.E. General Santander. Con un formato impreso, ver anexo 1. Y diez preguntas, y la opción de respuestas son SI (S), NO (N) e INDIFERENTE (I).

\section{Tabla 1. Preguntas Encuesta Diagnóstica}

\footnotetext{
1. Se puede establecer el aprendizaje de conceptos y métodos matemáticos asociados a la función cuadrática, mediante el uso de un simulador matemático.

2. Se puede mejorar el aprendizaje de los contenidos asociados a la función cuadrática si se tuviera un simulador matemático para graficar funciones.

3. Con un simulador matemático se puede obtener una mayor practicidad y aplicabilidad del aprendizaje, respecto a la opción de la lectura de libros y documentos.

4. Conocer y manejar el simulador matemático para resolver ejercicios de funciones cuadráticas aumentará el grado de motivación como estudiante de grado noveno.

5. Un simulador para resolver ejercicios de graficas de funciones cuadráticas mejorará el grado de autonomía en el aprendizaje no presencial de los estudiantes de grado noveno.

6. A través de un simulador matemático para resolver problemas y graficas de funciones cuadráticas, el estudiante podrá adquirir el desarrollo de habilidades de las tics.

7. Un simulador para la enseñanza de la función cuadrática convierte el computador en herramienta de ayuda para solucionar aspectos relacionados con la educación extra aula.

8. Un simulador matemático para la enseñanza de la función cuadrática, permite afianzar el proceso de desarrollo de educación a distancia mediante la técnica repetición práctica.

9. Un simulador matemático como estrategia de enseñanza para la función cuadrática resulta más llamativo y novedoso para los estudiantes que el usar exclusivamente textos impresos.

10. El simulador matemático facilitará la explicación de los conceptos relacionados con funciones cuadráticas.
}

Fuente: Autor

\section{Resultados}

Tabla 2.Resultados consolidados de la encuesta diagnóstica.

\begin{tabular}{lllllll}
\hline Pregunta & $\mathrm{Si}$ & $\%$ & No & $\%$ & Indiferente & $\%$ \\
\hline 1 & 37 & 92.5 & 3 & 7.5 & 0 & 0 \\
\hline
\end{tabular}




\begin{tabular}{lllllll}
\hline 2 & 40 & 100 & 0 & 0 & 0 & 0 \\
3 & 30 & 75 & 9 & 22.5 & 1 & 2.5 \\
4 & 16 & 40 & 20 & 50 & 4 & 10 \\
5 & 24 & 60 & 13 & 32.5 & 3 & 7.5 \\
6 & 39 & 97.5 & 1 & 2.5 & 0 & 0 \\
7 & 39 & 97.5 & 0 & 0 & 1 & 2.5 \\
8 & 38 & 95 & 2 & 5 & 0 & 0 \\
9 & 25 & 62.5 & 10 & 25 & 5 & 12.5 \\
10 & 23 & 57.5 & 15 & 37.5 & 2 & 5 \\
\hline \multicolumn{7}{c}{ Fuente: Autor }
\end{tabular}

Para la pregunta 1, se observa en la tabla que el $92.5 \%$ de los estudiantes establece el aprendizaje de conceptos asociados a la función cuadrática usando el simulador matemático. El 7.5 de la población no lo establece.

Pregunta 2: Se observa en la tabla que el $100 \%$ de los estudiantes afirma que puede mejorar su aprendizaje de los contenidos asociados a la función cuadrática si se tuviera un simulador matemático para graficar funciones.

Pregunta 3: Se observa que $92.5 \%$ de los estudiantes afirma que un simulador matemático se puede obtener mayor practicidad y aplicabilidad del aprendizaje respecto a la opción de libros y documentos. Un $7.5 \%$ no encuentra mayor practicidad y aplicabilidad utilizando el simulador.

Pregunta 4: Se observa que el 40\% de los estudiantes al conocer y manejar el simulador matemático para resolver ejercicios de funciones cuadráticas aumentará el grado de motivación como estudiante de grado noveno. Un 50\% afirma lo contrario. Y aun $10 \%$ le es indiferente la pregunta.

Pregunta 5: Podemos apreciar en la tabla que el $60 \%$ de los estudiantes afirma que un simulador para resolver ejercicios de graficas de funciones cuadráticas mejorará el grado de autonomía en el aprendizaje no presencial. Un 32.5\% afirma que no, y aun $7.5 \%$ le es indiferente la pregunta.

Pregunta 6: Se puede observar que el $97.5 \%$ de los estudiantes a través de un simulador matemático para resolver problemas y graficas de funciones cuadráticas, el estudiante podrá adquirir el desarrollo de habilidades de las tics. Un $2.5 \%$ afirma lo contrario.

Pregunta 7: Se puede apreciar en la tabla que un $97.5 \%$ de los estudiantes afirma que un simulador para la enseñanza de la función cuadrática convierte el computador en herramienta de ayuda para solucionar aspectos relacionados con la educación extra aula. Y aun $2.5 \%$ le es indiferente.

Pregunta 8. Se puede observar que un $95 \%$ de los estudiantes afirma que un simulador matemático para la enseñanza de la función cuadrática, permite afianzar el proceso de desarrollo de educación a distancia mediante la técnica repetición práctica. Un $5 \%$ está en desacuerdo. 
Pregunta 9. Podemos apreciar que a un $62.5 \%$ de los estudiantes un simulador matemático como estrategia de enseñanza para la función cuadrática resulta más llamativo y novedoso para los estudiantes que el usar exclusivamente textos impresos. El 37.5\% opina lo contario y a un $5 \%$ le es indiferente la pregunta.

Para la pregunta 10. Podemos visualizar en la tabla que un $57.5 \%$ de los estudiantes el simulador matemático le facilitará la explicación de los conceptos relacionados con funciones cuadráticas. Y un $37.5 \%$ opina lo contrario, a un $5 \%$ de la población le es indiferente.

\section{Encuesta de Satisfacción}

Se llevó a cabo una encuesta de satisfacción a 33 estudiantes de grado 904 JT, de la I.E. General Santander ya que en el momento de la de la aplicación de la encuesta se encontraban sólo este número de estudiantes presentes, con un formato impreso. Y diez preguntas, y la opción de respuestas son SI (S), NO (N) e INDIFERENTE (I).

\section{Tabla 3. Preguntas Encuesta de Satisfacción}

1.Recomendaría el simulador a sus compañeros de nivel

2.Fue complicado de usar el simulador matemático

3. ¿Disfruto el manejo del simulador?

4. La manera en que presenta la información fue clara y entendible.

5.Me brindo ayuda con cualquier problema que surgió

6. La organización de los menús o la información listada es lógica

7.Aprender a usar las funcionalidades del simulador fue difícil

8. Tiene una interfaz muy llamativa.

9. Tuve que solicitar asistencia para su manejo reiteradas veces

10. ¿Considera que la utilización del simulador matemático fue positiva para la comprensión del concepto de Función Cuadrática?

\section{Fuente: Autor}

\section{Resultados}

Tabla 4. Resultados consolidados de la encuesta de satisfacción.

\begin{tabular}{lllllll}
\hline Pregunta & $\mathrm{Si}$ & $\%$ & No & $\%$ & Indiferente & $\%$ \\
\hline 1 & 33 & 100 & 0 & 0 & 0 & 0 \\
2 & 4 & 12 & 29 & 88 & 0 & 0 \\
3 & 20 & 61 & 8 & 24 & 5 & 15 \\
4 & 23 & 70 & 4 & 12 & 6 & 18 \\
5 & 19 & 58 & 14 & 42 & 0 & 0 \\
6 & 27 & 82 & 5 & 15 & 1 & 3 \\
7 & 8 & 25 & 25 & 75 & 0 & 0 \\
8 & 23 & 67 & 6 & 18 & 4 & 15 \\
9 & 9 & 27 & 22 & 67 & 2 & 6 \\
10 & 22 & 67 & 7 & 11 & 4 & 12 \\
\hline
\end{tabular}

Fuente: Autor

Pregunta 1: Se puede observar en la tabla que el 100\% de los estudiantes recomendaría el simulador a sus demás compañeros de nivel. 
Pregunta 2: Se aprecia que el $12 \%$ de los estudiantes opina que si les fue complicado el uso del simulador matemático, un $88 \%$ responde que les pareció simple su manejo.

Para la pregunta 3. Apreciamos que un $61 \%$ disfrutó el uso del simulador matemático, un $24 \%$ opina que no y aun $15 \%$ le es indiferente el uso del simulador.

Pregunta 4: Analizamos que el $70 \%$ de la población encuestada afirma que la manera de presentación del simulador fue clara y entendible. Un $12 \%$ afirma que no y a un $12 \%$ le es indiferente la pregunta.

Pregunta 5: Destacamos en la tabla que un 58\% de la población encuestada afirmo que el simulador le brindo ayuda con cualquier inconveniente en que tuviera el estudiante al momento de graficar. Un $42 \%$ contesta que no le brindó ayuda.

Pregunta 6: Detallamos en la tabla que un $82 \%$ de la población opino que la organización de los menús en el simulador es lógica y comprensible, mientras que un $15 \%$ opina que no y aun $3 \%$ le fue indiferente la organización de los menús.

Pregunta 7: Describimos que un 25\% de la población afirma que usar las funcionalidades del simulador fue difícil, mientras que un $75 \%$ le fue fácil el manejo y uso del simulador.

Pregunta 8: Destacamos en la tabla que un 67\% de la población encuestada afirma que el simulador tiene una interfaz llamativa, un $18 \%$ opina que no le fue llamativa la interfaz y aun $15 \%$ le fue indiferente la interfaz.

Pregunta 9: Apreciamos que un 27\% de la población encuestada tuvo que pedir al docente asistencia reiteradas veces para el manejo del simulador, un $67 \%$ opina que no tuvo que pedir asistencia al docente y aun $6 \%$ le fue indiferente la asistencia del docente.

Pregunta 10: Destacamos en la tabla que un 67\% de los estudiantes le fue positivo el uso del simulador en la comprensión del concepto de función cuadrática, un $11 \%$ opina lo contario y a un $12 \%$ le fue indiferente el manejo del simulador matemático.

\section{Discusión de resultados}

El simulador Applet Descartes como herramienta virtual de aprendizaje, favoreció una nueva forma de aprendizaje para estudiantes de grado noveno de la I.E. General Santander, esto concuerda con Pérez (2013) "el simulador y su interfaz de comunicación proporcionan la realimentación que permite a los estudiantes reflexionar sobre el proceso de aprendizaje y conversar con el profesor y otros estudiantes".

Ahora bien el uso de simuladores compone un medio importante para desarrollar procesos de enseñanza - aprendizaje, esto va en consonancia con Méndez (2014)“Se concluye que el uso de simuladores mejora significativamente el aprendizaje, lo hace activo, participativo y promueve el interés de los estudiantes, se recomienda el uso de simuladores Java como herramienta didáctica y como estrategia de aprendizaje”. Y ratificado por Pérez (2011)“Los 
simuladores en la educación son una herramienta muy útil de aprendizaje. Facilitan al alumno y profesor el desarrollo del conocimiento con alto grado de autonomía, comprensión de situaciones reales."

\section{Conclusiones}

El uso del Applet Descartes versión 3.815 compone un medio importante para desarrollar procesos de enseñanza y aprendizaje de la matemática, dentro de éstas se destaca su fácil manejo, debido a que pueden realizar construcciones por medio de acciones fomentando prácticas de visualización. Otro aspecto significativo está en el rol del docente en su búsqueda bajo la concepción constructivista, el aprendizaje es significativo y relevante cuando la participación es activa por parte del estudiante en la construcción del conocimiento; se evidenció que los estudiantes llegaron a comprender eficientemente lo que estaban afirmando basándose en una figura representada en sus pantallas o mediante conjeturas lógicas. Se procuró disponer de recursos que facilitarán al estudiante la graficación y análisis de funciones; proporcionar un elemento tecnológico mediador entre el docente y el estudiante para una mayor practicidad, aplicabilidad del aprendizaje y aumentar la motivación del estudiante.

Todo lo anterior, hace evidente las respuestas a los interrogantes y al objetivo general de la investigación que apuntan a la necesidad de implementar una estrategia didáctica para el concepto de la Función Cuadrática a los estudiantes de grado noveno en la asignatura Algebra mediante un software educativo para la resolución de problemas y graficación.

Se evaluó la aceptación del uso y el manejo del software matemático Applet descartes versión 3.815, observando una gran favorabilidad y beneplácito del software por parte de la mayoría de los estudiantes de grado 904 de la Institución Educativa General Santander de Soacha-Cundinamarca.

\section{Bibliografía}

Bagur.,A.(2011).Matemáticas para todos. Obtenido de http://www.acmor.org.mx/descargas/mate106.pdf.

Balestrini A, M. (2006).Cómo se Elabora el Proyecto de Investigación. Venezuela: Servicio Editorial.

Cuevas V, C. (2000). ¿Qué es Software Educativo o software para la enseñanza? Departamento de Matemática Educativa del Centro de Investigación y de Estudios Avanzados del Instituto Politécnico Nacional. México. Obtenido de http://www.matedu.cinvestav.mx/ ccuevas/SoftwareEducativo.htm.

Bagur, A. (2011). Matemáticas para todos. Recuperado el 16 de abril de 2016, de: http://www.acmor.org.mx/descargas/mate106.pdf

Castillo A, C.(2014). Aprendizaje de adición y sustracción de números enteros a través de objetos físicos. Obtenido de: http://www.bdigital.unal.edu.co/47573/1/94442425\%20Cesar.pdf 
Díaz.J (2016). Aplicación PhET: estrategia de enseñanza aprendizaje de fracciones equivalentes. Revista Criterios, 23(1)111-123.Recuperado el 9 de mayo de 2017 de: http://www.umariana.edu.co/ojs-editorial/index.php/criterios/article/view/1193/1136

Educaplus. (2016). Dianas móviles suma. Recuperado el 15 de Diciembre de 2016 de: http://www.educaplus.org/game/dianas-moviles-suma

Gallardo, A. Hernández, A. (2007). Emergencia de los números enteros. Recuperado el 15 de Diciembre del 2016. http://www.matedu.cinvestav.mx/ maestriaedu/docs/asig5/Agallardo.pdf

Gaitán, V. (2013). Gamificación: el aprendizaje divertido. Recuperado el 15 de Diciembre de 2016 de: http://www.educativa.com/blog-articulos/gamificacion-el-aprendizaje-divertido/

Parisca, M. (2009). Coeficiente de la correlación de Pearson, media, moda y mediana. Recuperado el 15 de Diciembre de 2016 de: http://html.rincondelvago.com/coeficiente-de-lacorrelacion-de-pearson-media-moda-y-mediana.html

Pósito, R. (2012).El problema de enseñar y aprender ciencias naturales en los nuevos ambientes educativos (Tesis de Magister).Recuperado el 16 de abril de2016,de:http://sedici.unlp.edu.ar/bitstream/handle/10915/18190/Documento_completo. pdf? sequence $=3$

Fatela.M. (2009). Matemática guía no.10. Función Cuadrática. Obtenido de http://mfatela.blogspot.com/2009/07/matematica-funcion-cuadratica-guia-n-10.html.

FIB. (2005).Retro Informática. Obtenido de http://www.fib.upc.edu/retroinformatica/avui/simulacio.html.

Huamán V. H. (2005). Manual de Técnicas de Investigación. Conceptos y aplicaciones. Perú.

Mariño, D. (2008).El empleo de las NTIC en la formación pedagógica municipalizada.La Habana.

McFarlane, A. (1999) Educational use of ICT, Working Paper. Obtenido de http://bert.eds.udel.edu/ocde/quality/papers/papersframe.html.

MEN. (2013). Estándares básicos de competencias en matemáticas. Obtenido de http://www.eduteka.org/pdfdir/MENEstandaresMatematicas2003.pdf.

Méndez M., E.E. (2014). Simuladores java y aprendizaje del teorema trabajo-energía. Obtenido de http://recursosbiblio.url.edu.gt/tesiseortiz/2014/05/86/Mendez-Edner.pdf

Muñoz., J. (2013).Creación de escenas interactivas. el applet descartes. Obtenido de http://recursostic.educacion.es/newton/web/cursoavanzado/p1/index.html; 2013. 
Pérez C., C.A. (2013).Simulador para apoyar el proceso de enseñanza/aprendizaje de las operaciones matemáticas básicas en el tercer grado de educación primaria. Obtenido de http://jupiter.utm.mx/ tesis_dig/11682.pdf.

Pérez R., C.M. (2011). Fisim: simulador físico -matemático integrado a la plataforma de gestión del aprendizaje zera.

Pizarro, R. (2009). Las TICs en la enseñanza de las Matemáticas. Aplicación al caso de Métodos Numéricos. Tesis de Magíster en Tecnología Informática Aplicada en Educación. La Plata: Universidad Nacional de La Plata.

Salinas. H. (2010). Investigación Cualitativa y Cuantitativa. Obtenido de http://www.slideshare.net/himmel.salinas/1-enfoques-cualtitavito-y-cuantitativo.

UNESCO. (2012). Informe de seguimiento de la educación para todos en el mundo. París: UNESCO. 